\title{
Administration of ketamine for depression should be limited to psychiatrists
}

\author{
Brian Blum, DO, and Jessica Grey, MD
}

n $\mathrm{n}$ the modern-day practice of medicine, turf wars are more common than one may realize. Presently, an ongoing battle over who should be prescribing and administering ketamine for novel treatment uses is being waged among psychiatrists, anesthesiologists, family physicians, and emergency physicians. Whoever emerges victorious will determine whether psychiatric care is administered in a safe and cost-effective manner, or if it will merely benefit the bottom line of the prescriber. In this article, we discuss how ketamine may have a role for treatment-resistant depression (TRD), and why psychiatrists are uniquely qualified to prescribe and administer this medication for this purpose.

\section{New approaches to treatment- resistant depression}

Antidepressant medications, long the mainstay of depression treatment, have been shown to be safe and relatively equally effective, with varying tolerability. However, 33\% percent of patients do not achieve remission after 4 trials of antidepressant therapy. ${ }^{1}$ Most antidepressant efficacy studies report remission rates of $35 \%$ to $40 \%,{ }^{2}$ which means many patients require subsequent switching and/or augmentation of their treatment. ${ }^{3}$ The STAR ${ }^{*} \mathrm{D}$ trial demonstrated that after 2 adequate antidepressant trials, the likelihood of remission diminishes. ${ }^{4}$

After a patient's depression is found to be treatment-resistant, the onus of guiding treatment shifts away from the patient's primary care physician to the more specialized

psychiatrist. Few would question the suitability of a psychiatrist's expertise in handling complicated and nuanced mental illness. In order to manage TRD, psychiatrists enter a terrain of emerging novel therapies with rapid onset, different mechanisms of action, and parenteral routes of administration.

One such therapy is esketamine, the S-enantiomer of ketamine. The FDA approved the intranasal (IN) formulation of esketamine in March 2019 after the medication had been designated as a breakthrough therapy for TRD in 2013 and studied in 6 Phase III clinical trials. ${ }^{5}$ The S-enantiomer of ketamine is known to bind to the N-methylD-aspartate receptor stronger than the R-enantiomer. ${ }^{6}$ The mechanism of action of both stereoisomers on other receptors, such as opioid and alpha-amino-3-hydroxy-5-methyl-4-isoxazolepropionic acid (AMPA), is the focus of intensive research and remains to be fully elucidated, but initial studies indicate rapid neuroplasticity and synaptogenesis. ${ }^{7}$ What is clear is that this new intervention can provide relief to patients with TRD via a pharmacologically distinct mechanism. ${ }^{8}$

Ketamine may be administered intranasally, intravenously, or orally. A metaanalysis aimed at assessing differences in

LET YOUR VOICE BE HEARD

CURRENT PSYCHIATRY invites psychiatry residents to share their views on professional or clinical topics for publication in Residents' Voices. E-mail jbauer@mdedge.com for author guidelines.

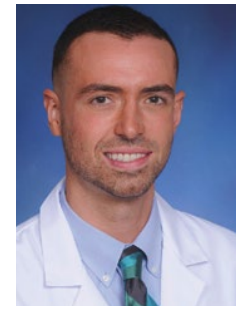

Brian Blum, DO

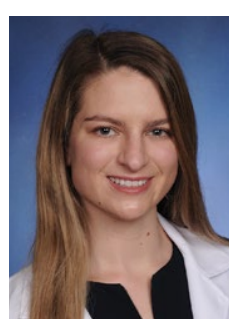

Jessica Grey, MD

Dr. Blum is a PGY-3 Psychiatry Resident, Department of Psychiatry, HCA Florida, Aventura Hospital and Medical Center, Aventura, Florida. Dr. Grey is a PGY-2 Psychiatry Resident, Department of Psychiatry, HCA Florida, Aventura Hospital and Medical Center, Aventura, Florida.

Disclosures

The authors report no financial relationships with any companies whose products are mentioned in this article, or with manufacturers of competing products. The views expressed in this article represent those of the authors and do not necessarily represent the official views of HCA Healthcare or any of its affiliated entities.

\section{Acknowledgment}

The authors thank Samuel Neuhut, MD, Chief of Psychiatry, Department of Psychiatry, HCA Florida, Aventura Hospital and Medical Center, Aventura, Florida, for his assistance with this article. doi: 10.12788/cp.0175 


\section{Clinical Point}

\section{Ketamine is being prescribed for psychiatric illnesses mainly by practitioners in fields other than psychiatry}

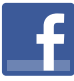

Discuss this article at www.facebook.com/ MDedgePsychiatry ketamine efficacy for depression based on route of administration have shown that both IV and IN ketamine are effective, though it is not possible to draw conclusions regarding a direct comparison based on available data. ${ }^{9}$ Despite several landmark published studies, such as those by Zarate et $\mathrm{al}_{1}^{10}$ IV ketamine is not FDA-approved for TRD.

\section{Why psychiatrists?}

Psychiatrists have been prescribing IN esketamine, which is covered by most commercial insurances and administered in certified healthcare settings under a Risk Evaluation and Mitigation Strategy program. ${ }^{5}$ However, anesthesiologists and emergency physicians have opened a crop of boutique and concierge health clinics offering various "packages" of IV ketamine infusions for a slew of mental ailments, including depression, anxiety, bipolar disorder, and posttraumatic stress disorder. ${ }^{11}$ Minimal investigation reveals that these services are being prescribed mainly by practitioners in fields other than psychiatry. Intravenous ketamine has long been used off-label as a treatment for depression not by psychiatrists but by practitioners of anesthesiology or emergency medicine. Although these clinicians are likely familiar with ketamine as an anesthetic, they have no foundation or expertise in the diagnosis and treatment of complex mood disorders. The FDA-approved indication for esketamine falls firmly in the realm of psychiatric treatment. Physicians who have not completed a psychiatry residency have neither the training nor experience necessary to determine whether a patient is a candidate for this treatment.

One potential adverse effect of ketamine is an emergence phenomenon, colloquially named a "K-hole," that can induce symptoms of psychosis such as disturbing hallucinations. Patients who have a history of psychosis need to be carefully evaluated for appropriateness to receive this treatment.

Furthermore, ketamine treatments administered by physicians who are not psychiatrists are billed not through insurance but mostly via private pay. A patient may therefore be charged $\$ 350$ to $\$ 1,000$ per infusion, to be paid out of pocket. ${ }^{11}$ Tally that up over the standard 6 to 12 initial treatment infusions, followed by maintenance infusions, and these patients with profound depression are potentially building up significant debt. Does this practice align with the ethical principles of autonomy, justice, beneficence, and nonmaleficence that all physicians swore to uphold? Will psychiatrists take a stand against the financial exploitation of a vulnerable group that is desperate to find any potential relief from their depression?

\section{References}

1. Hillhouse TM, Porter JH. A brief history of the development of antidepressant drugs: from monoamines to glutamate. Exp Clin Psychopharmacol. 2015;23(1):1-21.

2. Fava M, Rush A, Trivedi M, et al. Background and rationale for the Sequenced Treatment Alternatives to Relieve Depression (STAR*D) study. Psychiatr Clin North Am. 2003;26(2):457-494.

3. Gaynes BN, Rush AJ, Trivedi MH, et al. Primary versus specialty care outcomes for depressed outpatients managed with measurement-based care: results from STAR*D. J Gen Intern Med. 2008;23(5):551-560.

4. Gaynes BN, Warden D, Trivedi MH, et al. What did STAR*D teach us? Results from a large-scale, practical, clinical trial for patients with depression. Psychiatr Serv. 2009;60(11):1439-1445.

5. US Food and Drug Administration. Center for Drug Evaluation and Research. Esketamine clinical review. Published March 5, 2019. Accessed August 9, 2021. https://www.accessdata.fda. gov/drugsatfda_docs/nda/2019/211243Orig1s000MedR.pdf

6. Zanos P, Moaddel R, Morris PJ, et al. Ketamine and ketamine metabolite pharmacology: insights into therapeutic mechanisms. Pharmacol Rev. 2018;70(3):621-660.

7. Zanos P, Gould TD. Mechanisms of ketamine action as an antidepressant. Mol Psychiatry. 2018;23(4):801-811.

8. Kaur U, Pathak BK, Singh A, et al. Esketamine: a glimmer of hope in treatment-resistant depression. Eur Arch Psychiatry Clin Neurosci. 2021;271(3):417-429.

9. McIntyre RS, Carvalho IP, Lui LMW, et al. The effect of intravenous, intranasal, and oral ketamine/esketamine in mood disorders: a meta-analysis. J Affect Disord. 2020;276: 576-584.

10. Zarate CA Jr, Singh JB, Carlson PJ, et al. A randomized trial of an N-methyl-D-aspartate antagonist in treatment-resistant major depression. Arch Gen Psychiatry. 2006;63(8):856-864.

11. Thielking M. Ketamine gives hope to patients with severe depression. But some clinics stray from the science and hype its benefits. STAT+. Published September 18, 2018. Accessed August 5, 2021. www.statnews.com/2018/09/24/ketamineclinics-severe-depression-treatment/ 